\title{
Editorial
}

\section{Tumour-promoting role of EMT-inducing transcription factor ZEB1 in mantle cell lymphoma}

\author{
AE Sayan ${ }^{*, 1}$ \\ Cell Death and Differentiation (2014) 21, 194-195; doi:10.1038/cdd.2013.178
}

Epithelial-mesenchymal transition (EMT) is an embryonic trans-differentiation programme that is implicated in organ formation by facilitating the formation of highly motile cells with stem cell capabilities. ${ }^{1}$ Most tissues of cells derived from ectoderm or mesoderm retain certain elements of EMT and mesenchymal properties are their differentiation markers. On the other hand, cells of organs derived from endoderm only experience EMT and its reversal (mesenchymal-epithelial transition) at certain phases of development and become differentiated secretory epithelial cells. As de-differentiation is a common feature of cancer progression, acquisition of mesenchymal markers accompanied with the loss of epithelial features can be observed in most carcinomas and considered as the reason of cancer spread (metastasis). For the reasons that (1) more than $85 \%$ of all cancers are of epithelial origin and (2) epithelial (differentiated) and mesenchymal (undifferentiated) carcinoma cells are morphologically very different, EMT has been mostly studied in carcinoma setting. However, there is growing evidence suggesting that cancers of organs derived from mesoderm (e.g., hematopoietic malignancies and sarcomas) or ectoderm (e.g., glioblastomas and melanomas) also activate EMT programs to acquire an undifferentiated state and become resistant to conventional therapies. ${ }^{2}$ A good example of this is a recent article by Sanchez-Tillo et al. that investigated the role of EMT-inducing transcription factor ZEB1 in mantle cell lymphoma (MCL) progression. ${ }^{3}$

$\mathrm{MCL}$ is a B-cell malignancy and considered as the rarest form of non-Hodgkin lymphoma. ${ }^{4}$ Despite this statistical data, it is responsible for a significant portion of B-cell malignancy related mortality because it is an aggressive cancer with a continuous relapse pattern. ${ }^{5}$ In addition, most MCL patients show poor response to chemotherapy. The translocation of cyclin D1 to immunoglobulin heavy chain enhancer locus $t(11 ; 14)(q 13 ; q 32)$ is the genetic hallmark of MCLs; however Wnt, p53, Notch pathway aberrations and pro-survival gene overexpression ( $\mathrm{Mcl} 1$ and $\mathrm{Bcl} 2$ overexpression) are also commonly observed.

Sanchez-Tillo et al. investigated the expression of EMT-inducing transcription factor ZEB1 in a cohort of MCL patient samples. ${ }^{3}$ They found ZEB1 overexpression in half of the samples with a strong correlation with nuclear $\beta$-catenin presence (Wnt signalling active). In addition, the clinico-pathological analysis of patient data revealed ZEB1 positivity as a marker for shorter overall survival. This led them to investigate the mechanisms of ZEB1 upregulation and functional contribution of ZEB1 expression to the pathology of MCL. ZEB1 promoter has been previously reported (by the same group) to contain two TCF-LEF binding sites allowing $\beta$-catenin-TCF4 binding in colorectal cancer setting. ${ }^{6}$ They validated the presence of endogenous $\beta$-catenin on the respective DNA motifs of ZEB1 promoter by chromatin immunoprecipitation and also showed that ZEB1 mRNA levels are changing upon stimulation with Wnt-3a or knockdown of endogenous $\beta$-catenin using two different MCL cell lines. Following this observation, they also investigated if modulation of $\beta$-catenin signalling by chemical inhibitors such as salinomycin can alter ZEB1 expression. Salinomycin treatment, indeed, was able to decrease endogenous ZEB1 expression. These results mark the potential to control acquired ZEB1 expression by targeting Wnt pathway and inhibit its functional consequences in MCL.

The cellular consequences of ZEB1 overexpression in MCL have not been investigated, however, carcinomas expressing ZEB1 were shown to have canonical hallmarks of EMT such as increased invasiveness and metastasis, resistance to chemotherapy and decreased proliferation. ${ }^{7}$ Sanchez-Tillo et al. systematically tested all these aspects in MCL by knocking down ZEB1 or $\beta$-catenin using MCL cell lines. They found that ZEB1 downregulation decreases baseline cell viability and proliferation. The gene expression of ZEB1expressing cells indicated activation of survival and repression of cell death pathways as validated by increased levels of myc, cyclin D1, CENPF, Ki67, Bcl2, MCL1 and decreased p53, bax and PMAIP1 mRNAs. In addition, they also investigated whether ZEB1 expression confers resistance to chemotherapy. In line with their previous data regarding changes in baseline cell viability, ZEB1 downregulation increased sensitivity to commonly used chemotherapeutic drugs used in MCL treatment, such as Doxorubicin, Cytarabine and Gemcitabine but not Vincristine. The effect of ZEB1 knockdown on chemosensitization was also confirmed by pretreatment of MCL cell lines with Salinomycin.

EMT-induced chemoresistance has been described in different carcinoma models; however, the cellular mechanism of this phenomenon still remains to be clarified. ${ }^{8}$ The reasons

${ }^{1}$ Cancer Sciences Division, Somers Cancer Research Building, University of Southampton, Tremona Road, Southampton SO16 6YD, UK

${ }^{*}$ Corresponding author: AE Sayan, Cancer Sciences Division, Somers Cancer Research Building, University of Southampton, Tremona Road, Southampton SO16 6YD, UK. Tel: +44 (0) 238079 5762; Fax: +44 (0) 238079 5152; E-mail: a.e.sayan@soton.ac.uk 
for chemoresistance can be at least 3-fold. Cancer cells can alter drug influx/efflux to favour less chemotherapeutic agents in the cell (e.g., activate multidrug resistance proteins), they can activate efficient detoxification (e.g., by glutathione S-transferase) or enable efficient DNA repair to escape DNA damage-induced apoptosis. In this article, Sanchez-Tillo et al. investigated the regulation of drug influx/efflux pathways by ZEB1. Using two different MCL cell lines and validating by IHC in primary MCL samples, they showed that critical drug transporters are regulated by ZEB1 to favour survival of Wnt signalling active cancer cells.

The authors also extended their in vitro studies to animal models. Subcutaneous injection of Granta-519 cells expressing or silenced ZEB1 showed significantly different responses; ZEB1 silenced cells showing delays in tumour growth and enhanced sensitivity to Doxorubicin treatment. Finally, they explored whether salinomycin can synergize with Doxorubicin for an enhanced response to chemotherapy. Using established MCL cell lines and primary cells from MCL patients, they showed that sublethal doses of Salinomycin and Doxorubicin can synergize and effectively kill MCL cells. These results support their in vivo data and provide evidence

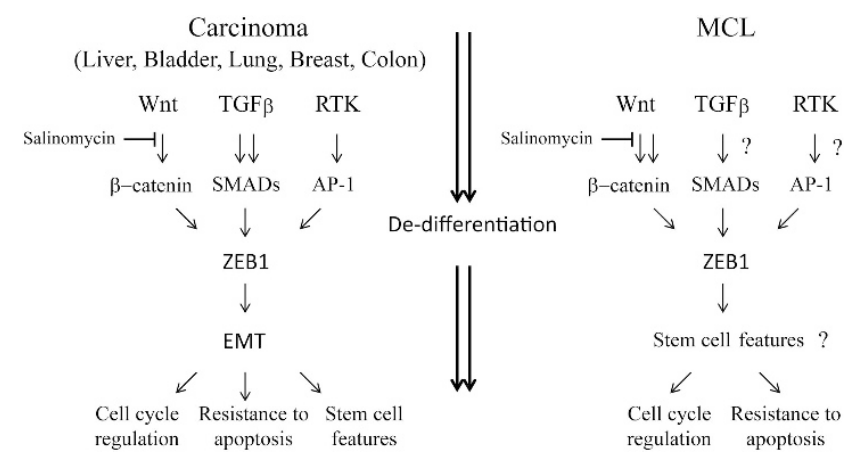

Figure 1 Role of EMT-inducing transcription factor ZEB1 in MCL. In carcinoma cells, activation of distinct signalling pathways such as $\operatorname{TGF} \beta$, Wnt and receptor tyrosine kinase (RTK) converges to ZEB1 for a fully de-differentiated and mesenchymal (EMT) phenotype. In this setting, TGF $\beta$ is considered as the main inducer of ZEB1. The functional consequences of ZEB1 activation is chemoresistance, cell cycle regulation and acquisition of stem cell properties. In MCL model, Wnt activation is the dominant signal however the consequences of ZEB1 induction (e.g., chemoresistance) remains the same. The involvement TGF $\beta$ and RTK signalling to ZEB1 activation and stem cell features of ZEB1-expressing MCL cells is yet to be assessed (and marked with '?') that $\beta$-catenin-activated ZEB1 expression contributes to tumour progression and chemoresistance (Figure 1).

What is next? A couple of things remained to be done and questions answered to make use of the full potential of these exciting findings. First of all, Sanchez-Tillo et al. did not show the clinico-pathological variables of their patient cohort. In recent years, anti-B cell-based immunotherapeutic regimens, such as the anti-CD20 antibody rituximab, gained attention as an alternative in the treatment of B-cell malignancies. The provision of detailed patient data may allow stratification of MCL cases and potentially mark subgroups that can or cannot be treated with chemo and/or immunotherapies. In addition, similar correlative studies have to be performed using independent patient cohorts to externally validate the clinical importance of ZEB1 in MCL and allow clinical translation. Another point to be clarified is the functional link between ZEB1induced alterations in drug influx/efflux and the actual presence of drugs in MCL cells. Genotoxic agents such as Doxorubicin can be imported through cell membrane by passive diffusion and effectively retained in the cell as DNA-bound form so they cannot be exported by drug transporters. This question can be easily answered due to the fluorescence nature of Doxorubicin using normal and transformed $B$ cells, in the presence and absence of ZEB1. Finally, activation of EMT pathways in carcinoma results in generation of malignant cells with stem cell properties. A critical question that remains to be answered is whether ZEB1-expressing MCL cells are de-differentiated enough to be considered as B-cell precursors.

As a follow-up of this work, it will be fascinating to explore if the tumour-promoting features of ZEB1 in MCL cells are also conserved in other malignancies, particularly in other mesoderm-derived tissues such as sarcomas or cancers such as hepatoblastomas that $\beta$-catenin activation is an early event in tumourigenesis.

\section{Conflict of Interest}

The author declares no conflict of interest.

1. Thiery JP et al. Cell 2009; 139: 871-890.

2. Caramel J et al. Cancer Cell 2013; 24: 466-480.

3. Sanchez-Tillo E et al. Cell Death Differ 2014; 21: 247-257.

4. Jares P, Colomer D, Campo E. Nat Rev Cancer 2007; 7: 750-762.

5. Herrmann A et al. J Clin Oncol 2009; 27: 511-518.

6. Sanchez-Tillo E et al. Proc Natl Acad Sci USA 2011; 108: 19204-19209.

7. Thomson S et al. Clin Exp Metastasis 2011; 28: 137-155.

8. Singh A, Settleman J. Oncogene 2010; 29: 4741-4751. 